\title{
Treatment of Advanced Prostate Cancer
}

by Theodore S. Thomas, MD \& Russell K. Pachynski, MD

\begin{tabular}{l} 
Washington \\
University in St.Louis \\
\hline School of MEdicine
\end{tabular}

\section{Gaps in our knowledge with respect to direct comparisons, combinations, and sequencing of these agents are currently being filled by numerous ongoing trials with the goals of improving both the quantity and quality of patients with prostate cancer.}

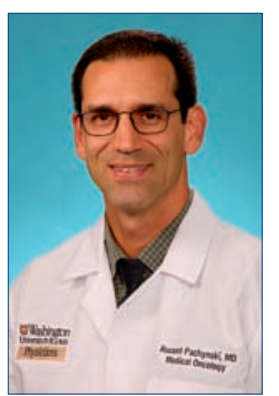

Theodore S. Thomas, MD, MPHS, is a staff Oncologist, St. Louis VA Medical Center and Russell K. Pachynski, MD, (above) is Assistant Professor, Division of Oncology, Washington University School of Medicine, St. Louis, Missouri.

Contact: rkpachynski@wustl.edu

\section{Abstract}

Advanced prostate cancer

is a heterogenous disease with multiple treatment options.

Patients with advanced disease are stratified based evidence of metastasis and sensitivity to hormone therapy. Men with hormone sensitive disease are treated with androgen deprivation therapy and possibly chemotherapy. The treatment options for men with castrate resistant disease are rapidly evolving with multiple recently approved treatment options. Determining the proper sequence and combination of these therapies remains a work in progress.

\section{Background}

Patients with prostate cancer (PCa) may present with localized (80\% of patients), locally advanced (10-15\% or patients), or distant metastatic disease. Advanced PCa defined by the presence of disease outside of the prostate, regional lymph nodes or pelvis. Patients with advanced disease are considered incurable but highly treatable with excellent long-term survival compared to most advanced malignancies. Advanced disease may present de novo at initial presentation (approximately 4-5\% of new diagnoses) or as progression following definitive therapy for localized disease.
PCa typically spreads through direct extension and hematogenously. Standard imaging consists of technetium bone scan and CT scans and should be considered in high risk patients ( $\mathrm{T} 1$ with prostatespecific antigen $[\mathrm{PSA}]>20, \mathrm{~T} 2$ with PSA $>10$, T3-T4, Gleason $>8$ or symptoms). Additionally, newer imaging modalities allow for increased sensitivity in detecting metastasis.

\section{Advanced PCa can be} categorized into two main entities: biochemical recurrence (BCR) after local therapy without radiographic evidence of metastasis (M0) or with evidence of metastasis (M1). Stage M0 potentially encompasses patients with micrometastatic disease not detectable with conventional imaging techniques. Most patients will eventually develop measurable metastatic disease; this timeframe is variable but usually measured in years. Post-prostatectomy PSA should be undetectable at 6 weeks postop and PSA increases may signify incomplete resection or BCR (if initially undetectable). Post radiation therapy, BCR is typically defined by a $>2.0 \mathrm{ng} / \mathrm{mL}$ rise in PSA above nadir. Several studies have pointed to improvements in metastasis-free survival with initiation of early treatment of BCR (usually defined as PSA $<0.5 \mathrm{ng}$ / $\mathrm{mL}$ ). However, the superiority of adjuvant (after surgery in high risk patients) versus salvage (at time of 
$\mathrm{BCR}$ ) radiotherapy is still an open question, with several ongoing trials addressing this topic. The decision to initiate treatment is a shared decision with the patient, involving discussion of both the benefits of treatment along with the risks of side effects. Importantly, novel imaging techniques with higher sensitivities may reclassify some of these M0 patients to M1 disease status. Systemic treatment is commonly initiated for men with M1 disease. Here we briefly review current treatment modalities for men with advanced PCa.

\section{Hormonal Therapy}

Androgens are crucial for proliferation, differentiation, and spread of PCa cells. ${ }^{1}$ Male androgens are produced predominantly in the testes (90\%) with the remainder produced in the adrenal glands. The hypothalamic-pituitary axis (HPA) is the key regulator of androgen synthesis. Luteinizing hormone-releasing hormone (LHRH) is released in a pulsatile fashion from the pituitary gland and acts on the hypothalamus to release luteinizing hormone (LH). LH stimulates testosterone production in the testes with a negative feedback loop to limit testosterone production.

Testosterone and the metabolite dihydrotestosterone (DHT) are required for prostate cell growth. Androgen deprivation results in an initial programmed cell death (apoptosis) in both normal and malignant prostate cells. The goal of systemic androgen deprivation therapy (ADT) for $\mathrm{PCa}$ is to suppress testosterone to castrate levels $(<50 \mathrm{ng} / \mathrm{dL})$. While the earliest described treatmentsurgical castration-is cost effective and safe, the adverse psychologic consequences have generally limited the use of this method. LHRH analogues (i.e. antagonists or agonists) are the most frequently used method of ADT. LHRH agonists (e.g. leuprolide) hyperstimulate the pituitary gland resulting in excess LH and FSH production, with resultant pituitary desensitization over 3-4 weeks. Downstream LH and testicular androgen production then ceases. However, the initial LHRH receptor stimulation temporarily increases testosterone production that may result in tumor growth. This transient "flare" may result in worsened symptoms including urinary obstruction, increased pain, and/or cord compromise in patients with spinal metastases. Overlapping treatment with an antiandrogen will reduce this risk and should begin 7-14 days prior to a LHRH agonist administration, with continuation for typically 30 days. Continuing antiandrogen administration with an LHRH analogue (i.e. maximal or combined androgen blockade) is effective, with improved clinical outcomes and overall survival (OS) in multiple studies.

LHRH receptor antagonists (e.g. degarelix) directly compete with endogenous LHRH in the pituitary gland. This results in rapid reduction of testosterone levels without the testosterone flare risk and is equally clinically effective. Potential advantages include reduction of FSH/ LH micro-surges, and decreased events in patient with cardiovascular comorbidities. All forms of ADT have similar side effects including fatigue, libido loss, cognitive effects, vasomotor symptoms, and metabolic effects.

\section{Anti-Androgens}

Anti-androgens are an additional method of hormone blockade. These therapies are used in combination with ADT and not approved for single agent use. First-generation anti-androgens include bicalutamide, flutamide and nilutamide. ${ }^{2}$ These compete with testosterone/DHT at the androgen receptor (AR) leading to reduced signaling through the AR. Eventually PCa cells become resistant to these therapies through various mechanisms, including AR overexpression/ amplification, mutations in ligand binding domains leading to constitutive signaling, and additional signaling pathways (e.g. glucocorticoid receptor, HGF/c-MET, etc.). Anti-androgen withdrawal can be an effective hormonal maneuver in which the anti-androgen is held for 4-6 weeks, although PSA responses occur in 10$15 \%$ of patients. Switching between first-generation anti-androgens may improve responses, however. Second-generation anti-androgens (e.g. enzalutamide, apalutamide/ARN-509) have been developed to combat this resistance. In addition to blockade of the AR, they additionally inhibit AR nuclear translocation, and association of AR with DNA. Enzalutamide is approved in the metastatic setting both before and after docetaxel use, while apalutamide is currently undergoing clinical trials.

\section{Androgen Biosynthesis Inhibitors}

Decreasing androgen biosynthesis is an additional tool used to block adrenal and intra-tumoral androgen synthesis. CYP17 is a key enzyme required for androgen synthesis and has been shown to be involved in the development of intra-tumoral androgen expression. ${ }^{3}$ Ketoconazole (in combination with hydrocortisone) is a non-specific CYP17 inhibitor and has been used 
extensively as a therapy in refractory PCa therapy. However, with side effects (e.g. fatal hepatoxicity, gastrointestinal symptoms), poor efficacy, and newer agents it now has a limited role in PCa treatment. Other therapies include estrogens, glucocorticoids, diethylstilbestrol (DES), all of which are rarely used due to availability of more effective therapies. Abiraterone, a selective CYP17 inhibitor, is approved in metastatic PCa in both the pre- and post-docetaxel settings. Typical side effects include electrolyte disturbances, fluid retention, adrenal insufficiency, and rarely hepatotoxicity.

\section{Hormone Sensitive Advanced PCa After Prior Local Therapy}

Patients treated with prior local therapy may have relapsed disease. The first step is to assess the disease burden. Asymptomatic patients with a rising PSA and no evidence of radiographic metastasis are said to have BCR. Asymptomatic men may initially be observed, as toxicity of systemic ADT may outweigh benefits depending on a variety of factors (e.g. comorbidities, performance status [PS], disease extent, etc.) and incorporating estimated life expectancy. Patients with oligometastatic disease recurrence may be considered for metastatectomy or radiation therapy (RT) to sites of disease. ${ }^{4}$ This may be associated with a survival benefit and role for these therapies continues to be evaluated. Most men with radiographic evidence of disease will need ADT. Whether ADT should be indefinite or intermittent remains an open question. ${ }^{5}$ Non-inferiority studies did not reach statistical significance but were associated with a better quality of life (QOL). However, the consensus remains that patients with high risk/high volume disease (typically defined as $\geq 4$ bony sites or any visceral disease) are more likely to benefit from continuous ADT.

\section{Hormone Therapy for Disseminated PCa}

Metastatic PCa is currently not curative, but most patients will have significant initial responses to therapy. Upfront ADT has been the standard of care for treatment naïve PCa, based on Huggins and Hodges seminal work in 1941. Patients with symptomatic disease or metastasis at high risk sites (i.e. spinal cord, visceral disease) should be treated promptly; symptoms from metastatic PCa can rapidly resolve within days to weeks if responsive to initial ADT, and thus it is important to consider this when evaluating PS and treatment decisions. The addition of a continuous anti-androgen to an LHRH analogue has been extensively studied, and meta-analyses have shown a small but significant benefit of combined androgen blockade. ${ }^{6,7}$ However, there is currently no recommendation for or against combined androgen blockade in this setting. ${ }^{8}$

\section{Chemohormonal Therapy}

More recently large clinical trials have demonstrated benefits of early initiation of chemotherapy concomitantly with ADT (chemohormonal therapy). Two major clinical trials, CHAARTED ${ }^{9}$ and STAMPEDE ${ }^{10}$ have evaluated the early use of docetaxel in hormone-sensitive PCa. The CHAARTED trial randomized 790 men with previously untreated metastatic PCa to ADT versus ADT with docetaxel (75 $\mathrm{mg} / \mathrm{m}^{2}$ every 3 weeks) for 6 cycles with a primary end point of OS. The primary endpoint was met with an OS benefit (57.6 vs. 44.0 months, HR 0.61) in the docetaxel arm. This study was stratified by disease burden (high versus low volume) with high volume disease defined by visceral metastasis and/or $\geq 4$ bone metastases. The OS benefit was predominantly seen in the high-volume disease group (49.2 vs 32.2 months, HR 0.60, p=0.0012). The chemotherapy group was associated with a higher rate of fatigue, neutropenic fever but overall was well tolerated by most men. An OS benefit of 10 months (81 versus 71) was also seen in the STAMPEDE trial with the addition of chemotherapy to ADT. Based on this evidence, patients with high volume, newly diagnosed or recurrent hormone therapy naïve metastatic $\mathrm{PCa}$ with adequate PS and organ function should be considered for chemohormonal therapy; this is widely accepted as the new standard of care therapy for these patients. The role of chemohormonal therapy for patients with N0/1 and M0 disease is still being evaluated as the risk/ benefit ratio in low volume disease patients is less clear.

\section{Castrate Resistant PCa}

PCa that grows despite castrate levels of testosterone $(<50 \mathrm{ng} / \mathrm{dL})$ is classified as castrate resistant (CRPC). This progression can present as increasing PSA, new metastasis, or progression of existing metastatic disease sites. There are multiple proposed mechanisms of resistance, including overexpression or activation of AR, de novo synthesis of androgens within the tumor, or non-androgen driven 
growth pathways. Several therapies have been recently Federal Drug Administration (FDA) approved that are effective at treating CRPC.

\section{Anti-Androgens in CRPC}

The addition of antiandrogens to ADT is associated with improved disease control and OS benefit in CRPC. Recent data has shown the superiority of the second-generation enzalutamide to first-generation bicalutamide. The STRIVE Trial was a double-blind, phase II study of 396 men with both metastatic and non-metastatic CRPC comparing enzalutamide versus bicalutamide in combination with ADT. ${ }^{11}$ Enzalutamide reduced the risk of progression or death by 76\% (hazard ratio $0.24,95 \% \mathrm{CI} 0.18$ to $0.32, \mathrm{p}<.001$ ) with a median progression-free survival (PFS) of 19.4 versus 5.7 months, compared to the bicalutamide arm. These data, amongst others, suggest that use of enzalutamide should be favored over first-generation antiandrogens in the setting of CRPC. However, the use of enzalutamide in this setting has not yet been FDA approved, nor incorporated into the National Comprehensive Cancer Network (NCCN) guidelines as standard practice. In other studies of mCRPC, the use of enzalutamide was evaluated both before and after taxane-based chemotherapy. Enzalutamide was first demonstrated to be beneficial after taxane-based chemotherapy. The AFFIRM study included 1,199 men with metastatic CRPC (mCRPC) previously treated with 1-2 chemotherapy regimens who were then randomly assigned (2:1) to enzalutamide vs placebo. ${ }^{12}$ Treatment with enzalutamide was associated with a 4.8 month benefit in OS (18.4 vs. 13.6 months, HR 0.63, p <0.001) and improved QOL (43\% vs. $18 \%, \mathrm{p}<0.001)$. The drug was overall well tolerated but was associated with fatigue, diarrhea, and rarely seizures. In the PREVAIL trial, chemotherapynaïve $\mathrm{mCRPC}$ patients were randomized to enzalutamide vs placebo. Men receiving enzalutamide had improved radiographic PFS at 12 months (65\% vs. 14\%, HR 0.19, $\mathrm{p}<0.001)$, 29\% risk reduction for death (72\% vs. 63\%, HR $0.71, \mathrm{p}<0.001)$, and delayed chemotherapy by 17 months with estimated median OS at 32.4 months and 30.2 months for enzalutamide and placebo, respectively. ${ }^{13}$ Side effects were more common in the enzalutamide group including fatigue, constipation, and arthralgia. Most recently, apalutamide was approved for use by the FDA in men with non-metastatic CRPC. In a randomized, placebo controlled Phase III trial (the SPARTAN trial), treatment with apalutamide resulted in an over 2 year delay in the development of metastatic disease. Treatment was well tolerated, and there was a trend- albeit nonsignificant- towards improvement in OS in the setting of still maturing data and follow up.

\section{Androgen Biosynthesis Inhibitors in CRPC}

The development of selective CYP17 inhibitors has largely replaced older agents. Abiraterone is the only FDA approved selective CYP17 inhibitor and has been evaluated and approved in both the pre- and postchemotherapy mCRPC setting. In the COU-AA-301 trial, 1195 men with mCRPC who received prior docetaxel chemotherapy were randomized (2:1) to abiraterone 1000 $\mathrm{mg}$ plus prednisone (5 $\mathrm{mg}$ twice daily) versus placebo plus prednisone. ${ }^{14}$ The primary end point was met with a 3.9 month OS improvement (14.8 vs. 10.9 months, HR 0.65, $\mathrm{p}<0.001)$. Improvements in PSA progression $(10.2$ vs. 6.6 months) and PFS (5.6 vs. 3.6 months) were also noted. Abiraterone has also been shown to be effective prior to chemotherapy. In the COU-AA-302 trial, 1088 chemotherapy-naïve mCRPC patients were randomized (1:1) to receive abiraterone or placebo, both with prednisone. Treatment with abiraterone was associated with an improvement in median OS (not reached vs 27.2 months, HR $0.42, \mathrm{p}<.001$ ) with an improvement in PFS as well. ${ }^{15} \mathrm{~A}$ longer term follow-up study confirmed the OS improvement (34.7 vs 30.3 months, HR 0.81, $\mathrm{p}=0.0033) .{ }^{16}$ The side effect profile was similar in both studies with notable adverse events occurring in the abiraterone group including fluid retention, hypertension, electrolyte and liver enzyme abnormalities.

\section{Cytotoxic Chemotherapy for Metastatic PCa}

Cytotoxic chemotherapy plays an important role in the management of metastatic PCa. There are three FDA approved chemotherapies: mitoxantrone, docetaxel, and cabazitaxel. Mitoxantrone is a synthetic anthracenedione that inhibits DNA topoisomerase II, and was the first chemotherapy approved in advanced PCa. Mitoxantrone in combination with hydrocortisone was compared to hydrocortisone alone in the CALGB 9182 trial; approval was based on improvements in QOL and bone pain. ${ }^{17}$ However, mitoxantrone failed to demonstrate an OS advantage, with median OS 12.3 versus 12.6 months compared with hydrocortisone alone. Largely due to 
newer approved agents that carry improvements in OS, mitoxantrone use in PCa has declined over the last decade.

Docetaxel is a semi-synthetic taxane, and is the most commonly used chemotherapy in PCa. The approval of docetaxel in advanced PCa was originally based on a significant survival benefit compared with mitxanterone. ${ }^{18}$ The TAX-327 trial randomized 1006 men $(1: 1: 1)$ to docetaxel $75 \mathrm{mg} / \mathrm{m}^{2}$ every 3 weeks, docetaxel $30 \mathrm{mg} / \mathrm{m}^{2}$ weekly ( 5 of 6 weeks), or mitoxantrone $12 \mathrm{mg} / \mathrm{m}^{2}$ every 3 weeks. Docetaxel showed improved outcomes with median OS of 18.9 months (every 3 weeks) versus 17.4 months (weekly) versus 16.5 months in the mitoxantrone group. Docetaxel was also associated with an improvement in QOL. Side effects were similar to mitoxantrone, but docetaxel was associated with higher frequency of neutropenia and neutropenic fever.

Cabazitaxel is a second-generation taxane chemotherapy developed to overcome resistance in patients previously treated with docetaxel. In the TROPIC trial, cabazitaxel was shown to have an OS benefit (15.1 vs. 12.7 months) in comparison to mitoxantrone in mCRPC patients who progressed after docetaxel. ${ }^{14}$ However, there were increased rates of neutropenia (82\% vs 58\%), neutropenic fever (8\% vs $1 \%)$ and hypersensitivity reactions in the cabazitaxel arm.

\section{Radiation Therapy in Metastatic CRPC}

Approximately $90 \%$ of advanced PCa patients will develop bone metastases. Radiation therapy is an important therapeutic option for of bone disease. Patients with isolated, painful bone lesions may be treated with palliative external beam radiation. Additionally, oligometastatic disease may be treated to slow progression. Systemically administered, bone targeted radiopharmaceuticals can be used for control of bone predominant disease. Radium-223 is an alpha particle emitting agent approved for the treatment of mCRPC patients with symptomatic bone lesions, without visceral disease. Patients in the ALSYMPCA trial receiving radium-223 (vs. placebo) had an improvement in OS (14.9 vs. 11.3 months) as well as significant improvements in skeletal- and pain-related outcomes. ${ }^{20}$ Treatment with radium-223 alone has been shown effective in comparison to placebo; however, there are several ongoing studies investigating combinations with other approved agents in mCRPC.

\section{Immunotherapy in Metastatic CRPC}

Immunotherapy aims to augment the immune response against progressing cancers. Sipuleucel-T is an FDA-approved autologous dendritic cell vaccine that acts by enhancing T-cell responses. Treatment with sipuleucel-T $(n=341)$ produced a significant improvement in OS (median 25.8 vs. $21.7 \mathrm{mos}$, HR 0.78, $\mathrm{p}=0.032$ ) compared with a similar leukocyte product placebo $(n=171)$; however, there was no significant improvement in PFS, and only 2.6\% of patients in the sipuleucel-T arm had PSA declines of $\geq 50 \% .{ }^{21}$ Prostvac is a PSA/vaccinia-based vaccine currently being evaluated, with impressive improvements in OS in phase II trials. Unfortunately, the registration Phase III trial was recently reported to be negative. However, there are several ongoing trials of Prostvac in combination with checkpoint inhibitors. - Ipilimumab (anti-CTLA-4 monoclonal antibody) has been evaluated in two recently reported Phase III studies in mCRPC patients. While there was minimal improvement in OS with borderline significance $(p=0.052)$ in the first reported trial in post-

chemotherapy patients receiving radiation, ${ }^{22}$ a second trial in pre-chemotherapy trials was also unfortunately negative. ${ }^{23}$ Combination studies with ipilimumab, as well as other checkpoint inhibitors (e.g. anti-PD-1, anti-PD-L1) are actively being evaluated in the mCRPC setting.

\section{Supportive Care}

A key aspect of the management of advanced $\mathrm{PCa}$ is adequate supportive measures. Men with PCa can develop symptoms from metastatic disease sites (pain, fractures), from prior therapies (incontinence, impotence, radiation proctitis), and from current therapies. Controlling these symptoms is paramount to ensuring good QOL in the setting of incurable disease.

Management of complications of bone metastases is important. Those with pain should be prescribed sufficient analgesic medications, to ensure relief of cancer related symptoms. Systemic therapies may help with symptoms in newly diagnosed patients. Bone strengthening agents used in metastatic PCa consist of 
bisphosphonates (e.g. zoledronic acid) and osteoclast inhibitors (e.g. denosumab), and can significantly reduce the rates of skeletal related events. The use of bone strengthening agents in patients with hormone sensitive $\mathrm{PCa}$, however, has not been demonstrated to reduce pain, time to first skeletal related events, or survival. In patients with osteopenia on ADT, denosumab should be considered to prevent progression of bone mineral density loss. ${ }^{24,25}$

\section{Conclusions}

Since the approval of docetaxel in 2004, the available therapies that improve survival and QOL for patients with advanced PCa have rapidly expanded. Novel AR-targeted therapies, chemotherapies, and immunotherapies are promising areas of research and will continue to be evaluated. Gaps in our knowledge with respect to direct comparisons, combinations, and sequencing of these agents are currently being filled by numerous ongoing trials with the goals of improving both the quantity and quality of patients with PCa.

\section{References}

1. Kluth LA, Shariat SF, Kratzik C, et al. The hypothalamic-pituitarygonadal axis and prostate cancer: implications for androgen deprivation therapy. World journal of urology. 2014;32(3):669-676.

2. Wong YNS, Ferraldeschi R, Attard G, de Bono J. Evolution of androgen receptor targeted therapy for advanced prostate cancer. Nat Rev Clin Oncol. 2014;11(6):365-376.

3. Montgomery RB, Mostaghel EA, Vessella R, et al. Maintenance of intratumoral androgens in metastatic prostate cancer: a mechanism for castration-resistant tumor growth. Cancer research. 2008;68(11):44474454.

4. Ost P, Bossi A, Decaestecker K, et al. Metastasis-directed therapy of regional and distant recurrences after curative treatment of prostate cancer: a systematic review of the literature. European urology. 2015;67(5):852863.

5. Hussain M, Tangen CM, Berry DL, et al. Intermittent versus continuous androgen deprivation in prostate cancer. The New England journal of medicine. 2013;368(14):1314-1325.

6. Akaza H, Hinotsu S, Usami M, et al. Combined androgen blockade with bicalutamide for advanced prostate cancer: long-term followup of a phase 3, double-blind, randomized study for survival. Cancer. 2009;115(15):3437-3445

7. Samson DJ, Seidenfeld J, Schmitt B, et al. Systematic review and metaanalysis of monotherapy compared with combined androgen blockade for patients with advanced prostate carcinoma. Cancer. 2002;95(2):361-376. 8. Loblaw DA, Virgo KS, Nam R, et al. Initial hormonal management of androgen-sensitive metastatic, recurrent, or progressive prostate cancer: 2006 update of an American Society of Clinical Oncology practice guideline. J Clin Oncol. 2007;25(12):1596-1605.

9. Sweeney CJ, Chen YH, Carducci M, et al. Chemohormonal Therapy in Metastatic Hormone-Sensitive Prostate Cancer. N Engl J Med. 2015;373(8):737-746.

10. James ND, Sydes MR, Clarke NW, et al. Addition of docetaxel, zoledronic acid, or both to first-line long-term hormone therapy in prostate cancer (STAMPEDE): survival results from an adaptive, multiarm, multistage, platform randomised controlled trial. Lancet. 2016;387(10024):1163-1177. 11. Penson DF, Armstrong AJ, Concepcion R, et al. Enzalutamide Versus Bicalutamide in Castration-Resistant Prostate Cancer: The STRIVE Trial. Journal of Clinical Oncology. 2016;34(18):2098-2106.

12. Scher HI, Fizazi K, Saad F, et al. Increased survival with enzalutamide in prostate cancer after chemotherapy. The New England journal of medicine. 2012;367(13):1187-1197.

13. Beer TM, Armstrong AJ, Rathkopf DE, et al. Enzalutamide in metastatic prostate cancer before chemotherapy. The New England journal of medicine. 2014;371(5):424-433.

14. de Bono JS, Oudard S, Ozguroglu M, et al. Prednisone plus cabazitaxel or mitoxantrone for metastatic castration-resistant prostate cancer progressing after docetaxel treatment: a randomised open-label trial. Lancet (London, England). 2010;376(9747):1147-1154

15. Ryan CJ, Smith MR, de Bono JS, et al. Abiraterone in metastatic prostate cancer without previous chemotherapy. The New England journal of medicine. 2013;368(2):138-148

16. Ryan CJ, Smith MR, Fizazi K, et al. Abiraterone acetate plus prednisone versus placebo plus prednisone in chemotherapy-naive men with metastatic castration-resistant prostate cancer (COU-AA-302): final overall survival analysis of a randomised, double-blind, placebo-controlled phase 3 study. The Lancet Oncology. 2015;16(2):152-160.

17. Kantoff PW, Halabi S, Conaway M, et al. Hydrocortisone with or without mitoxantrone in men with hormone-refractory prostate cancer: results of the cancer and leukemia group B 9182 study. J Clin Oncol. 1999;17(8):2506-2513. 18. Tannock IF, de Wit R, Berry WR, et al. Docetaxel plus prednisone or mitoxantrone plus prednisone for advanced prostate cancer. The New England journal of medicine. 2004;351(15):1502-1512.

19. De Bono JS H-BA, Kin CS. Phase III non-inferiority study of cabazitaxel 20 $\mathrm{mg} / \mathrm{m} 2$ versus $25 \mathrm{mg} / \mathrm{m} 2$ in patients with metastatic castration-resistant prostate cancer previously treated with docetaxel2016.

20. Parker C, Nilsson S, Heinrich D, et al. Alpha emitter radium-223 and survival in metastatic prostate cancer. The New England journal of medicine. 2013;369(3):213-223

21. Kantoff PW, Higano CS, Shore ND, et al. Sipuleucel-T immunotherapy for castration-resistant prostate cancer. The New England journal of medicine. 2010;363(5):411-422

22. Kwon ED, Drake CG, Scher HI, et al. Ipilimumab versus placebo after radiotherapy in patients with metastatic castration-resistant prostate cancer that had progressed after docetaxel chemotherapy (CA184-043): a multicentre, randomised, double-blind, phase 3 trial. The Lancet Oncology. 2014;15(7):700712.

23. Beer TM, Kwon ED, Drake CG, et al. Randomized, Double-Blind, Phase III Trial of Ipilimumab Versus Placebo in Asymptomatic or Minimally Symptomatic Patients With Metastatic Chemotherapy-Naive Castration-Resistant Prostate Cancer. J Clin Oncol. 2017;35(1):40-47.

24. Fizazi K, Carducci M, Smith M, et al. Denosumab versus zoledronic acid for treatment of bone metastases in men with castration-resistant prostate cancer: a randomised, double-blind study. Lancet (London, England). 2011;377(9768):813822.

25. Smith MR, Halabi S, Ryan CJ, et al. Randomized controlled trial of early zoledronic acid in men with castration-sensitive prostate cancer and bone metastases: results of CALGB 90202 (alliance). J Clin Oncol. 2014;32(11):1143-1150

\section{Disclosure}

None reported. 